\title{
EFFECTS OF SHOCK WAVES ON COMBUSTION EFFICIENCY OF SUPERSONIC FLAMES
}

\author{
Albert Ratner and James F. Driscoll \\ University of Michigan \\ Dept. of Aerospace Engineering/FXB Building \\ 1320 Beal Âve., University of Michigan \\ Ann Arbor, MI 48109 \\ aratner@engin.umich.edu and jamesfd@engin.umich.edu
}

\begin{abstract}
Combustion efficiency was measured for a supersonic, non-premixed, jet-like flame. The flame was stabilized along the axis of a Mach 2.5 wind tunnel and wedges were mounted on the sidewalls in order to produce oblique shocks that interacted with the flame. Data was taken with air at stagnation temperatures of 284 $\mathrm{K}$ and $497 \mathrm{~K}$, at various fuel flow rates. Efficiency was measured using a solid state hydrogen gas detector that analyzed exit flue gas. It was observed that moving the wedges to different down-stream locations affected the visible flame length, but had only minor impact on the combustion efficiency in both air temperature case. The oblique shocks produced about a $8 \%$ reduction in combustion efficiency at 284 $\mathrm{K}$, with wedge position changing the fuel flow level needed to see certain characteristics. The reduction in efficiency and matching reduction in flame length are due to the shock waves. The shock waves cause radial inflow and outflow that allows some of the fuel to escape unburned. In the $497 \mathrm{~K}$ case, this same flow pattern causes a decrease when the flame is small and is not large enough to heat a significant portion of the air around it; but, this same flow pattern boosts combustion efficiency when the core of the flame is sufficiently large.
\end{abstract}

\section{Introduction}

Shock-wave-flame interaction is a common occurrence in any supersonic combustion environment. Because of this, it is important to understand both how this interaction occurs and the implications it has for both the flow field and the chemical kinetics. It is also important to note that supersonic flames have extremely high strain rates, are often lifted, and operate in what would normally be thought of as a near blowout state. Greater understanding of this flame state improves both knowledge of the physical world as well as providing verification for computer models that are the essential next step in scramjet development. Previous work to understand this problem was done by Yoon, et al. $(1,3)$ and Driscoll, et al. (2). By examining supersonic flame properties with minimal shock interaction, they were able to better understand the stability, blowout, and similar characteristics of the flame in a shock-free regime. 
This was followed by the work of Huh, et al. $(4,5,6)$ who measured flame length, choking limits, blow-out limits, and pressure distributions in flames interacting with strong oblique shocks. Huh found that shocks always decrease flame length, but he found that the amount by which the flame length changes varies with the shock down-stream position and fuel flow rate. Huh reasoned that the shock wave introduces a radial inflow of air into the flame, and that this greatly improves mixing. But, since Huh was unable to measure how much of the fuel was burned, he was unable to determine how beneficial this mixing might be. Experiments were done with cold (284 K stagnation temperature) and hot (497 K stagnation temperature) co-flowing air, and it was found that Huh was correct concerning the mixing that takes place, but the benefits to the flame are temperature and fuel flow rate dependent.

\section{Procedure}

The experimental apparatus is the same as that used by Huh, et al. $(5,6)$. A schematic of the experiment is shown in Figure 1. The air is regulated such that there is a constant flow of $1.07 \mathrm{~kg} / \mathrm{s}$ of air through the combustor at a Mach number of 2.5 . The hydrogen fuel enters along the axis of the combustor and is frictionally choked, so that the fuel tube exit Mach number is unity. This means that the Mach number for fuel is always 1 , even though the exit velocity and density changes as the driving pressure is increased or decreased. This allows the variation of the fuel flow rate from $0.78 \mathrm{~g} / \mathrm{s}$ up to $1.56 \mathrm{~g} / \mathrm{s}$. The fuel tube diameter is $0.7 \mathrm{~cm}$ inside and $2.54 \mathrm{~cm}$ outside. This large lip helps stabilize the flame, even though the flame itself is lifted several centimeters off of the tube. The flame varies in length from about 15 to $25 \mathrm{~cm}$ long.

The wedges that generate the oblique shocks that traverse the flame are mounted to the sidewalls of the combustor and have a slope of 10 degrees. They were inserted at two downstream locations, $2.8 \mathrm{~cm}(4 \mathrm{~d})$ and $5.95 \mathrm{~cm}(8.5 \mathrm{~d})$. Samples were taken with a probe that has an inner diameter of $1.3 \mathrm{~cm}$. The sample probe was located on the axis of the flue pipe and about 2 meters downstream of the combustor. This is about 300 fuel tube diameters downstream of the flame, and the flow can be assumed to be well mixed. The sampled gas was then run through a condenser coil that was kept in an ice bath so as to remove the water vapor. The gas was then run through 2 water filters (to confirm a dry reading), a flowmeter (to maintain the correct flow rate for the detector), and finally to the solid state hydrogen gas detector.

The detector (Toximet, made by Enmet Corp.) operates by catalyzing hydrogen, and since the catalyst is coupled to a silicon substrate, an electrical current varies with the hydrogen concentration. The detector determines the fraction of hydrogen that is present in the flue gas in about 1 to 2 minutes. The sensor is temperature compensated and both temperature and hydrogen gas calibrations are performed by the manufacturer (Enmet Corp.). A calibration was also performed with the detector in the actual laboratory configuration. Known amounts of fuel flowed through the fuel injector and mixed with the known air flow in a non-reacting situation, were then sampled, and a hydrogen concentration reading was given by the detector. It was found that the detector has both good repeatability and linearity over the range from 200 PPM to 20,000 PPM of $\mathrm{H}_{2}$, as shown in Figure 2. 


\section{Data Analysis}

The data that is generated by the detector is based on the dry fraction, so it is necessary to convert the dry gas reading into true mole fractions. The relation between the measured (dry) mole fraction and the actual mole fraction is:

$$
\frac{\chi_{\text {actual }}}{\chi_{\text {initial }}}=\frac{\left(\frac{1}{\chi_{\text {initial }}}-\frac{3}{2}\right)}{\left(\frac{1}{\chi_{d r y}}-\frac{3}{2}\right)}
$$$$
\eta_{\text {comb }}=\frac{\chi_{\text {initial }}-\chi_{\text {actual }}}{\chi_{\text {initial }}}
$$

Xinitial is the known ratio of the number of moles/sec of hydrogen injected to the moles/sec of air and hydrogen, prior to combustion. The combustion efficiency can then be calculated from the initial and actual mole fractions.

\section{Results For Stagnation Temperature of $284 \mathrm{~K}$}

Figure 3 shows the combustion efficiency of the three flame configurations at $T_{0}=284 \mathrm{~K}$, namely no shock waves, wedges placed at $x=4 d$ and wedges placed at $x=8.5 \mathrm{~d}$, as described above. All three cases show a linear, or nearly linear, improvement in combustion efficiency as the fuel mass flow rate is increased. Adding more fuel causes several things to happen, the most easily seen is that the flame becomes larger. The larger the flame, the more air it can heat and the longer the fuel spends in the hot flame core. Also, since the air next to the flame is preheated for a longer period of time, the likelihood of combustion continuing when a large parcel of nearby air is entrained into the flow is much higher for a larger flame than a smaller flame. This means that less of the fuel can escape unburned for a larger flame, and hence the combustion efficiency increases. This explains the upward trend that is seen in all three graphs. But looking at Figure 3, there is an almost uniform drop in efficiency when wedges, and hence shock waves, are introduced into the flow.

The explanation for this is that the shocks create a radial inflow of air from outside the flame followed by a radial outflow of this air mixed with some of the fuel and combustion products. The inflow of air from outside the flame boundary cools the region of the flame that it comes in contact with; although, the larger the flame, the more this air has been preheated and the smaller the impact. This air cools the flame and stops combustion, making this zone visible by an absence of light behind the shock. Then this cooled mixture undergoes a radial outflow, carrying away unburned fuel. This explains the decrease in efficiency, but there is more structure to the graphs in Figure 3. To understand this, an examination of the flame is required to detail the states that are present.

Figure 4 shows a diagram of the flame. The first thing to note is that due to the highly lifted nature of this flame, it is mostly premixed. This explains one phenomenon, that when shock waves pass through the flame, they extinguish it. But afterwards, it is observed to re-light. The flame is a premixed mass of fuel and air, and if it is hot enough and contains enough fuel, it will re-light. To ease analysis, various states of the flame will 
be labeled and then flow structure in each state can be compared to the efficiency data. The state where the fuel flow rate is low enough so that the flame burns all of the fuel before it reaches the shock waves will be called state 1 . As fuel flow rate is increased in this regime, the flame height grows smoothly. State 2 is the condition for which there is enough fuel for the flame to fully re-light downstream of the shock and grow smoothly with increasing fuel flow rate. Between these two states, is what will be referred to as a partially re-lit state. In the partially re-lit state, unsteady processes cause variation in the amount of unburned fuel just downstream of the shock waves. Some of the time there is enough fuel for the flame to re-light, and sometimes there is not. This is seen as a flicker, where the flickering gets stronger and more frequent as the fuel flow rate is increased. So, how do these states affect the combustion efficiency?

States 1 and 2 share the same flame growth and efficiency increase trends with the no wedge case. The larger the flame becomes, the smaller the impact of the wedges becomes, which is seen in Figure 3. Another way to visualize the physical process is to think of states 1 and 2 as the same state, and the partially re-lit state as a separate affect that has been superimposed in a sub-region of that state. It is believed that the cause of the partially re-lit state is the extinguishing of the flame, and doesn't affect states 1 and 2 in any way. As for the structure of the partially re-lit state, the graph of efficiency should show an abrupt change as state 1 is departed, followed by an increase in efficiency as flicker becomes more frequent and the flame is able to re-light more often. This is exactly what is seen in Figure 3. Also, as the wedges are moved downstream, it is expected that the end of state 1 and beginning of state 2 should both shift. This is because it takes more fuel to create a larger flame that will reach to the location of the shock waves. The shift should also be about the same amount (of fuel flow) and in the direction of increasing fuel. This can be seen in Figure 3.

\section{Results For Stagnation Temperature of $497 \mathrm{~K}$ and Comparison}

The results for the high stagnation temperature ( $497 \mathrm{~K}$ ) air flow cases are shown in Figure 5. Again, three cases were studied, one for flow without wedges, one with wedges near (4.0d) the fuel tube, and one with wedges farther away (8.5d). The expected result from extrapolating the cold air cases is that the combustion efficiency for all three cases should be larger for the higher stagnation temperatures, and it is. It is expected that the no wedge case will show both an increase in efficiency and a flattening of its slope as the efficiency approaches $100 \%$. This expected result is seen in Figure 6. For the wedge cases, it is expected that they will be very similar to each other and that the efficiency difference between wedges and the no wedge case should shrink as temperature is increased and the mixing induced by the shock waves becomes more beneficial. What is not expected in raising the temperature to $497 \mathrm{~K}$ is the amount of improvement in the combustion efficiency. This improvement, occurring at high fuel flow rates, can be so large as to yield a better combustion efficiency than even the no wedge case.

Another effect that can be linked to the improvement is that the deviation from the no wedge case is dependent on wedge position. The farther upstream the wedges, the greater the impact. This indicates that there is a preferential wedge location to minimize efficiency loss at low fuel flow rates, and a different location for mixing-enhanced efficiency increases at large fuel flow rates. These effects can be seen in Figure 5. 


\section{Conclusions}

The combustion efficiencies of supersonic hydrogen-air flames has been quantified and it has been shown that the effects of shock waves on the combustion efficiency are very dependent on both the flame size and the stagnation temperature of the air. For a small flame or one in cold air, air inflow into the flame due to shocks can hurt efficiency by cooling the flame and leave more fuel unburned. Conversely, for very high fuel flow rates and heated air, shock induced mixing can be helpful by bringing more air into a premixed but fuel-rich environment. Shocks also help to stabilize flames in certain configurations. All of these things combine to demonstrate that use of wedges or walls designed to create shock waves in scramjets may be beneficial, but it is realized that shock waves also cause detrimental losses in stagnation pressure and thrust, so the optimum design of shock locations and strengths is a challenge.

\section{Acknowledgments}

Support for Albert Ratner was made possible by a Doctoral Fellowship from the Association Francois-Xavier Bagnoud. The support of the Association is greatly appreciated. Partial support for this work was provided by the Advanced Propulsion Division, Wright Patterson AFB, AFOSR contract DOD G-F49620-95-1-0115, monitored by Dr. J. M. Tishkoff and the University of Michigan.

\section{References}

1. Yoon, Y., Donbar, J. M., and Driscoll, J. F., "Blowout Stability Limits of a Hydrogen Jet Flame in a Supersonic, Heated, Coflowing Air Stream," Combust. Sci. Technol. Vol 97, pp. 137-156, 1994.

2. Driscoll, J.F., Huh, H., Yoon, Y., and Donbar, J. M., "Measured Lengths of Supersonic Hydrogen-Air Flames-Compared to Subsonic Flame Lengths and Analysis," Combustion and Flame Vol 107, pp. 176-186, 1996.

3. Yoon, Y., Donbar, J., Huh, H., and Driscoll, J. F., "Measured Supersonic Flame Properties: Heat-Release Patterns, Pressure Losses, Thermal Choking Limits," ALAA J. of Prop. and Power Vol 12, No. 4, pp. 718-723, 1996.

4. Huh, H. and Driscoll, J. F., "Measured Effects of Shock Waves on Supersonic Hydrogen-Air Flames," AIAA Paper 96-3035, Jul. 1996.

5. Huh, H. and Driscoll, J. F., "Shock-Wave-Enhancement of the Mixing and the Stability Limits of Supersonic Hydrogen-Air Flames," $26^{\text {th }}$ Symp. (Inter) Combust. Combustion Inst., pp. 2933-2939, 1996.

6. Huh, H., "An Experimental Study of Supersonic Hydrogen-Air Flames for Scramjet Applications," Doctoral Thesis, University of Michigan, 1996. 


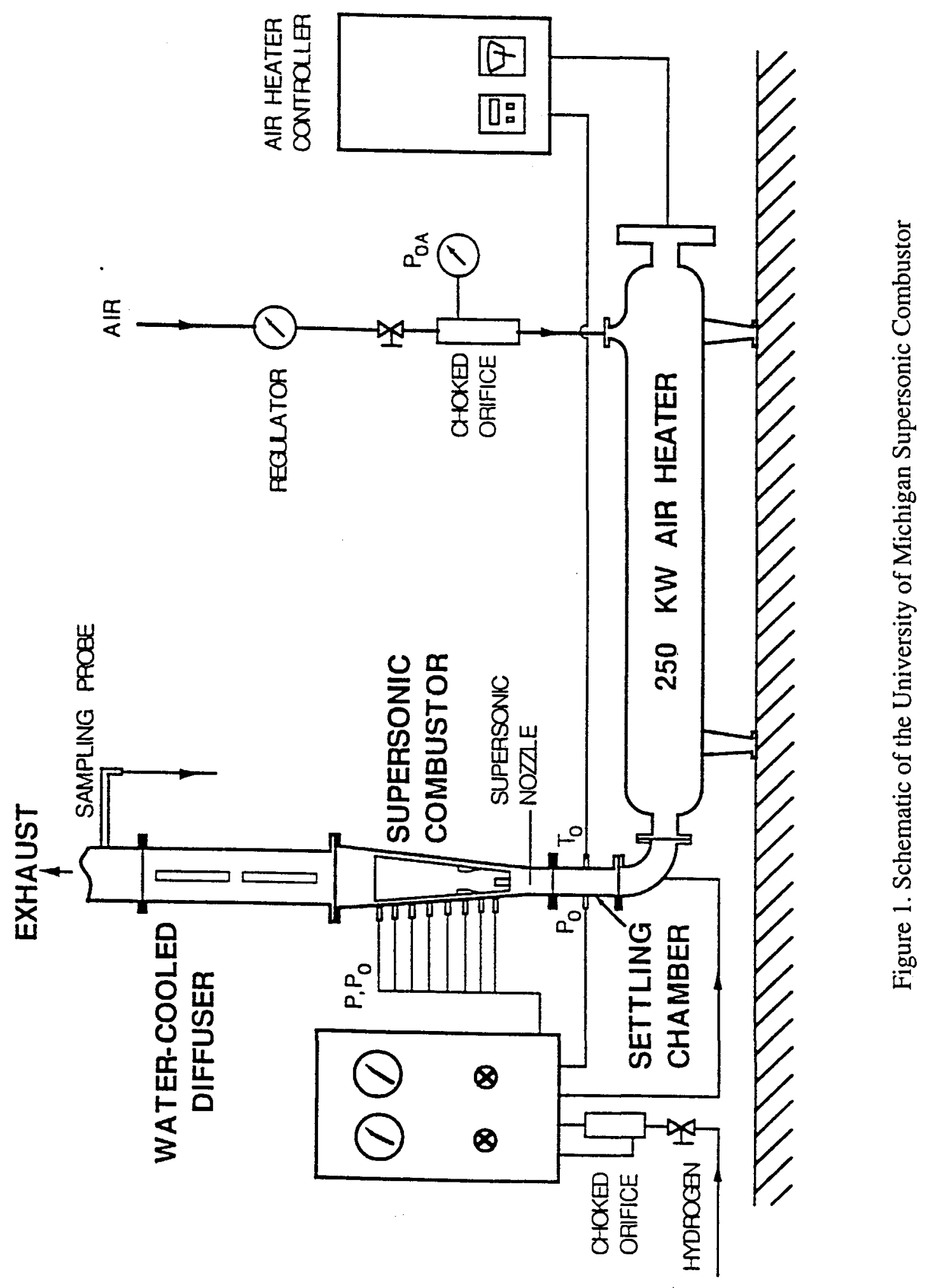




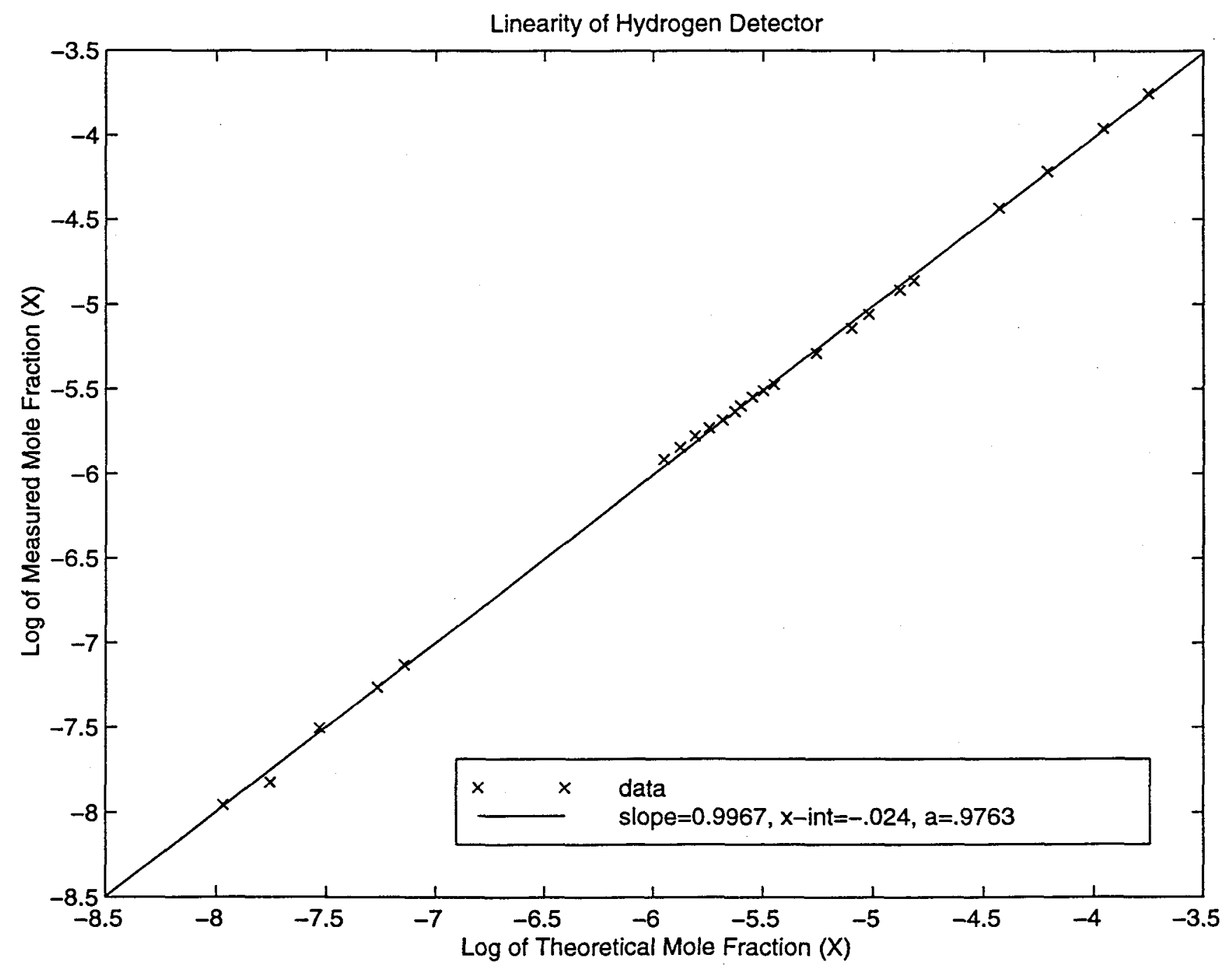

Figure 2. Linearity of Hydrogen Detector 


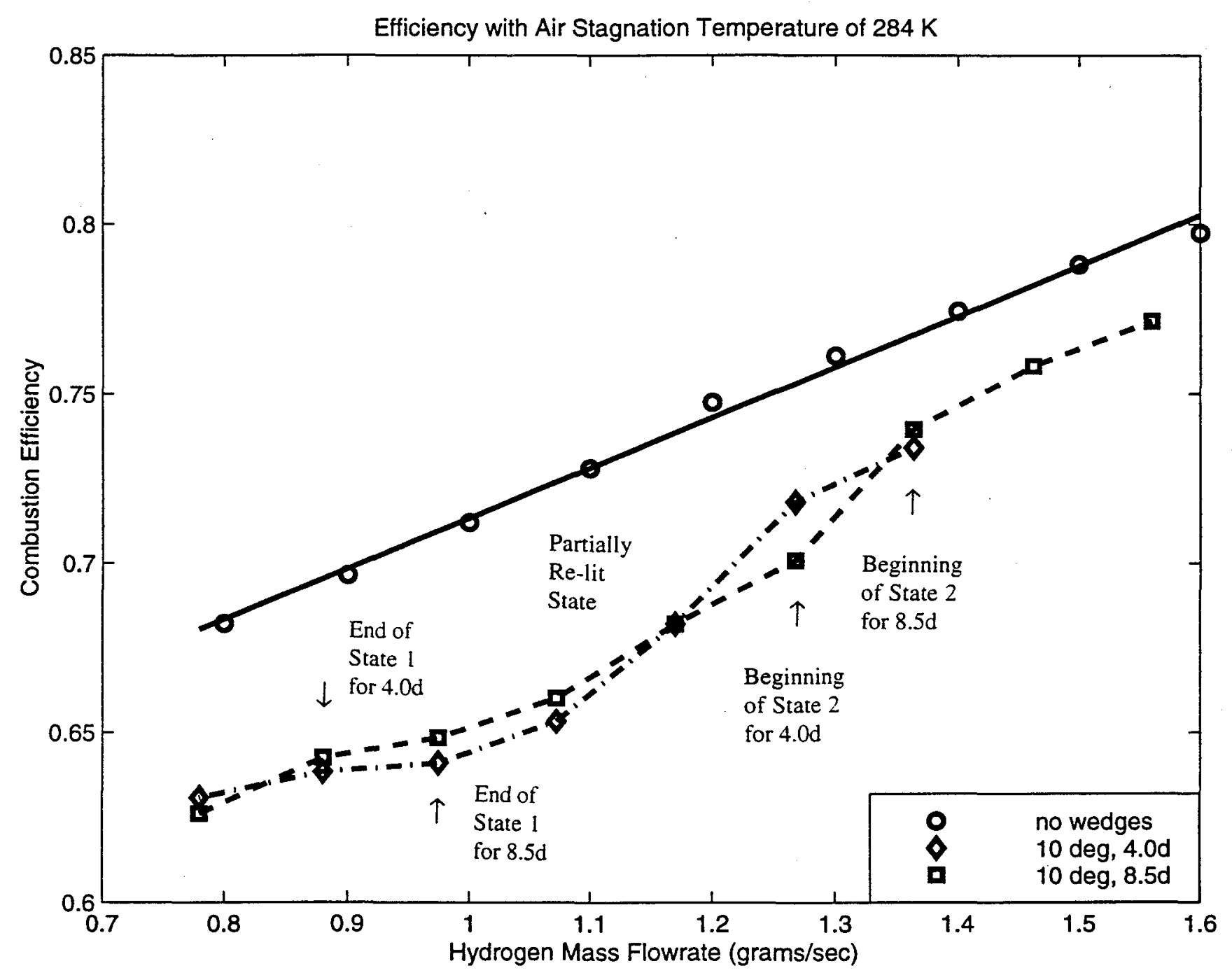

Figure 3. Measured Combustion Efficiencies of Supersonic Flames at Air Stagnation Temperature of $284 \mathrm{~K}$ 


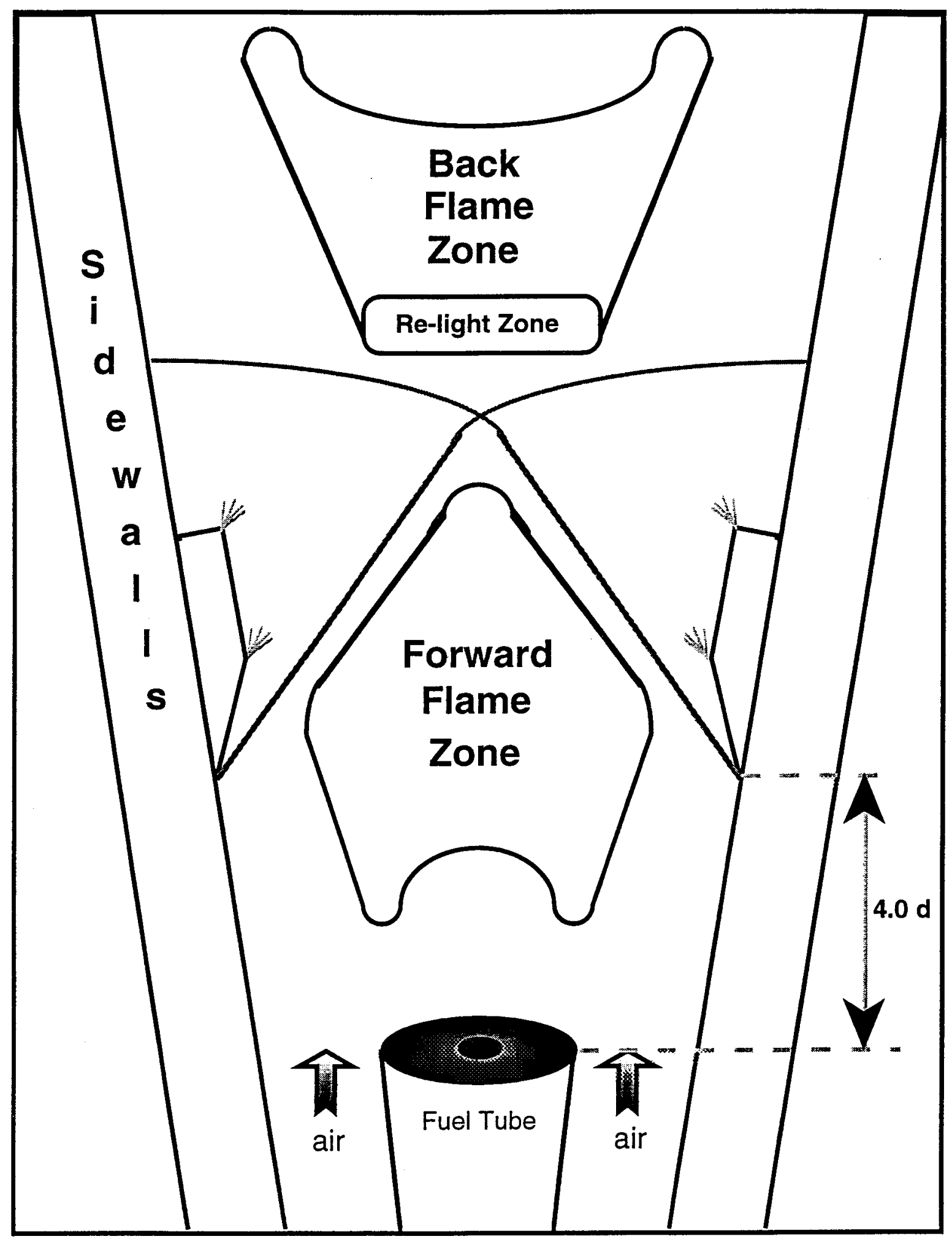

Figure 4. Diagram of Flame Structure in the Supersonic Combustor 


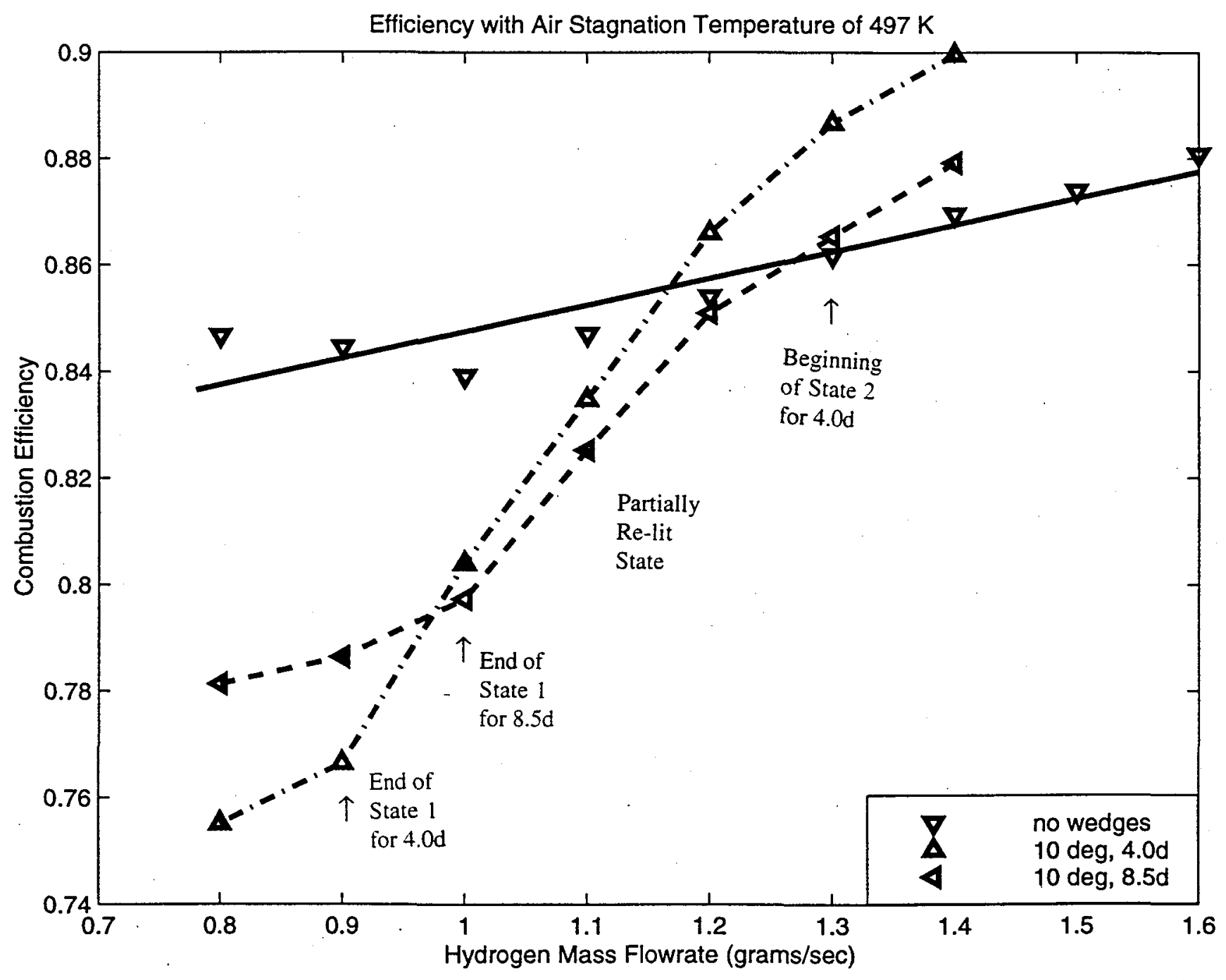

Figure 5. Measured Combustion Efficiencies of Supersonic Flames at Air Stagnation Temperature of $497 \mathrm{~K}$ 


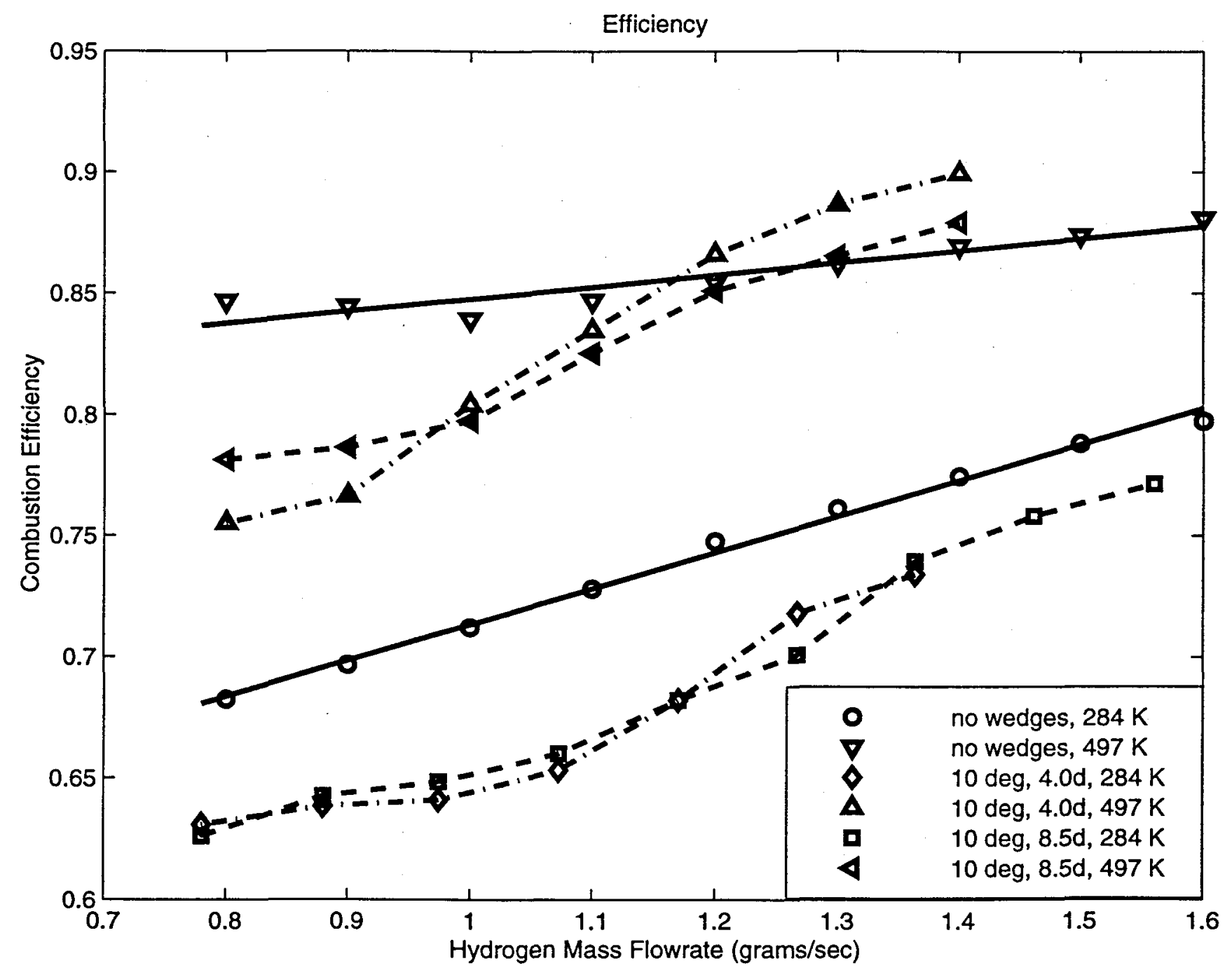

Figure 6. Measured Combustion Efficiencies for Both Air Temperatures 